\title{
Preparing to Teach Mathematics Pathways beyond 16
}

Cathy Smith, Jennie Golding and Nicola Bretscher

Open University, UK; University College London Institute of Education, UK

This special issue addresses two questions of national and international interest: Is there anything distinctive about learning to teach the kinds of advanced mathematics that students meet towards the end of compulsory education? If so, how does this affect the priorities and the design of secondary teacher development programmes? The four papers, submitted through the external peer-review process under the overall editorship of Anne Watson, arise from projects instigated at UCL Institute of Education (UCL IOE). This institution is notable in teacher education for its combination of large postgraduate courses and a research group that informs and critiques that provision. These papers show how the field of mathematics education can develop from such opportunities to analyse existing pedagogies, innovate in teaching, and research those processes. They focus on interactions between teachers' post-16 mathematics teaching and their professional learning, in the context of England's pre-university curriculum. Two pathways are considered: the well-established 'A-level' mathematics course that prepares for mathematics-rich undergraduate degrees, and the newer 'Core Maths' course that aims for students to become 'users of mathematics' in authentic contexts.

The background to this special issue comes from two discussions. The 2016-17 seminars Developing Pedagogy in Advanced Mathematics were organised by the editors as part of the Further Mathematics Support Programme. We sought to create an academic discussion amongst initial teacher educators and researchers, who often work in institutional silos, raising the profile of preparation for teaching post-16 and championing cross-institution provision. Participants, including all authors, collaborated in comparing, justifying and critiquing the approaches embedded in their own courses. The ideas in these papers owe much to that critique from other researcherpractitioners.

The second discussion arose while presenting aspects of these projects at the 2017 IMA conference Mathematics Education beyond 16: Pathways and Transitions, which assembled practitioners and researchers, from schools through to universities. A feature of this conference was discussion of how teaching approaches or emphases designed to meet the needs for one group or age of students became more widely influential. The perspectives of educators in Higher and Further Education have 
contributed to our thinking about how initial teacher education functions in the overlap between 'school' and 'university/college' practices of learning and of mathematics.

The first two papers in this issue focus on teacher development in relation to post-16 mathematics. Both papers examine teacher accounts of learning to teach for a specific curriculum, and the symbiosis with other teaching. They consider how intended institutional support has materialised, and affected what teachers do in the classroom and what they feel capable of.

The paper by Smith and Golding reports a longitudinal cross-case study of five teachers who had been allocated A-level classes in the first two years of their career. It shows how well-prepared beginning teachers can harness pre-university teaching to develop in ways that complement their other teaching. These teachers came to better appreciate connections within mathematics, the need for development of rigour and deep conceptual foundations, as well as deepening their own mathematical knowledge. However, the paper highlights the dissipation of mechanisms - even mandatory ones - that support professional development in A-level teaching.

In contrast, Golding, Smith and Blaylock offers a snapshot at a critical moment during the introduction of Core Maths, just as initial support for early adopters was withdrawn. The context here is professional learning of established teachers, where the third author is a lead practitioner. The collaboration with researchers led to framing an enquiry that covered both policy enactment and knowledge of how a new curriculum changed teaching and teachers. Using teacher accounts of the challenges and opportunities for reflection in learning to teach Core Maths, this paper offers an optimistic vision of teachers' capacity to change their practice in ways that align with underlying beliefs. Notably, the paper shows how the curriculum promotes student agency in the classroom and maps a consequential transition in how the teacher conceptualises mathematics and the pedagogic demands of such teaching.

The second pair of papers focus on pedagogy within initial teacher education. The first considers design at the tactical level of specifying connections and coherence within a new course, while the second addresses the technical level of matching course goals with user experience given the affordances of a particular mode of teaching, in this case synchronous online learning.

Smith and Bretscher report a self-study of developing design principles for a large course, Advanced Mathematics Teaching in Early Career (AMTEC), offered via national funding to interested early career teachers. The paper identifies challenges of balancing participants' attention between mathematics and classroom pedagogy, theorises related issues and offers the notion of 'key pedagogic messages' as a tactic for shifting this attention. It is both a practical paper, detailing the 
operationalising of these pedagogic messages, and a call for more principled consideration of how classroom approaches are modelled in teacher education settings.

Within AMTEC, Golding and Bretscher then consider how design solutions should change with mode of learning. Their study reveals how learning outcomes differed between the original, face-to-face version of the AMTEC course and a parallel synchronous online version. This provoked reflections on, and developments of, aspects of webinar pedagogy, notably their contingent formative assessment and their pedagogy for classroom tool use. They conclude that mode-specific challenges to participants' learning of subject knowledge could be resolved, but it is more challenging to design for the growth of pedagogical content knowledge.

As editors, we see certain themes arising from the interaction of these papers. Firstly, they show how the design and the medium of a course - whether in teacher education or in undergraduate education - can impact the learning made available in ways which transcend the sum of the subject matter to which students are exposed. They underline how teachers' mathematical knowledge and subject pedagogic knowledge develop together, in a longitudinal and non-linear fashion. International comparisons of teacher education highlight differences in the emphases placed on these. We argue that post-16 mathematics represents a critical site for research and for mathematics teacher development, precisely because both subject knowledge and pedagogic skills are in question. The papers on initial teacher education show the need for teacher educators to be purposeful in shifting attention between mathematics and pedagogy, while those on teacher development show how related interactions occur over many months, informed as much by analysis of student needs and questions as by mathematical enquiry. Secondly, we note the combined evidence of the benefits of mentoring and external programmes for challenging and supporting teachers beyond initial qualification - and in particular for targeted development of teaching post16. This supports proposals to extend English teachers qualifying period to three years, and more importantly, to create a framework of recognised and incentivised career development. Finally, the papers challenge a common model of teachers in deficit, showing that good subject knowledge and pedagogic content knowledge can be the basis for collaborative and/or semi-autonomous development, given some time and access to additional expertise or reflection with an informed other. 